\title{
Evaluation of Microscopic Extension in High-grade Glioma Using Macropathology: Determination of Optimal Clinical Target Volume Margins for Radiotherapy
}

Shulun Nie shandong cancer hospital

Yufang Zhu

shandong cancer hospital

Jia Yang

Shandong Cancer Hospital: Shandong Cancer Hospital and Institute

Tao Xin

Shandong Qianfoshan Hospital

\section{Song Xue}

shandong cancer hospital

Xianbin Zhang

shandong cancer hospital

Jujie Sun

shandong cancer hospital

Dianbin Mu

shandong cancer hospital

\section{Yongsheng Gao}

Shandong Cancer Hospital: Shandong Cancer Hospital and Institute

\section{Zhaoqiu Chen}

Shandong Cancer Hospital: Shandong Cancer Hospital and Institute

\section{Xingchen Ding}

shandong cancer hospital

Jinming Yu

shandong cancer hospital

Man Hu ( $\sim$ human5770@163.com )

Shandong Cancer Hospital and Institute https://orcid.org/0000-0003-4409-286X

\section{Research}


Keywords: High-grade glioma, macropathology, microscopic extension, predictive model, clinical target volume, radiotherapy

Posted Date: February 12th, 2021

DOI: https://doi.org/10.21203/rs.3.rs-194344/v1

License: (1) This work is licensed under a Creative Commons Attribution 4.0 International License. Read Full License 


\section{Abstract}

Introduction: One of the most difficult steps of radiotherapy is to determine the clinical target volume (CTV) based on microscopic extension (ME). In this study, we performed a consecutive macropathologic analysis to assess ME in high-grade glioma (HGG) to determine appropriate CTV margins for radiotherapy.

Material and methods: The study included $30 \mathrm{HGG}$ patients with tumors located in non-functional areas, and supra total resection was performed. The ME distance from the edge of the tumor to the microscopic tumor cells surrounding brain tissue was measured. Associations between the extent of ME and clinicopathological characteristics were evaluated by multivariate linear regression (MVLR) analysis. An ME predictive model was developed based on the MVLR model. Meanwhile, in order to validate the feasibility and safety of this model, we prospectively recruited another 30 HGG patients in a 1:1 ratio to receive guideline-based radiotherapy (RT) or model-based RT. The overall response rate (ORR) was evaluated during a follow-up of 14 months.

Results: Between June 2017 and July 2019, 652 pathologic slides were analyzed. The mean ME distance was $1.70 \mathrm{~cm}$ (range, 0.63 to $2.87 \mathrm{~cm}$ ). The MVLR analysis identified that pathologic grade, subventricular zone (SVZ) contact and $0^{6}$-methylguanine-DNA methyltransferase (MGMT) methylation, isocitrate dehydrogenase (IDH) mutation and 1p/19q co-deletion status were independent variables predicting ME (all $P<0.05$ ). A multivariable prediction model was developed as follows: $\mathrm{Y}_{\mathrm{ME}}=0.672+0.513 \mathrm{X}_{\mathrm{Grade}}{ }^{+}$

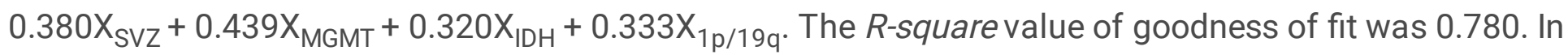
our validation cohort, after a mean follow-up of 7.65 months, patients in the model RT group had a higher ORR than those in the guideline RT group $(66.7 \%$ vs. $20 \%, P=0.01)$.

Conclusion: ME was heterogeneously distributed across different grades of gliomas according to the tumor location and molecular marker status, which indicated that CTV delineation should be individualized. The model could predict the ME of HGG, which may help clinicians determine the CTV for individual patients.

\section{Introduction}

High-grade glioma (HGG) is the most commonly diagnosed primary brain tumor, and has a remarkable tendency to infiltrate the surrounding brain tissue. To protect brain function, gross total resection through surgery becomes almost impossible. Therefore, radiotherapy (RT) has become the main treatment for HGG patients. In National Comprehensive Cancer Network (NCCN) guidelines, for the delineation of a clinical target volume (CTV), a margin accounting for subdiagnostic tumor infiltration, of $1-2.5 \mathrm{~cm}$ for HGG in terms of the volumetric expansion of the gross target volume (GTV) is recommended. This is empirically determined, based on data demonstrating that over $80 \%$ of recurrences occur within a $2 \mathrm{~cm}$ margin of the contrast-enhanced lesion on computed tomography (CT) or magnetic resonance imaging (MRI) [1-4]. Thus far, this evidence is just indirect and inadequate. Direct evidence for CTV delineation 
should be provided by the infiltration margin of the tumor. However, assessing the microscopic extension (ME) in HGG is challenging.

Pathology, as the gold standard of diagnosis, can precisely evaluate the ME of tumors. Unfortunately, it is difficult to obtain an adequate surgical margin for HGG, since the tumor is generally removed piecemeal under microscopy. Therefore, few previous studies have revealed the extent of the peripheral infiltration margin of glioma cells (GCs). Through autopsy, Burger et al. [5] found that GCs with a high migratory capability could infiltrate beyond $2 \mathrm{~cm}$ of the contrast-enhanced rim on CT. This two-dimensional study is limited to one brain histological section per case and lacks detailed data for CTV delineation. Two biopsied studies [6,7] further showed that GCs could deeply infiltrate the contralateral hemisphere, which revealed that HGG had a potential tendency to invade further. However, this limited information does not provide precise evidence for target delineation.

With the development of genomics, $0^{6}$-methylguanine-DNA-methyltransferase (MGMT) promotes methylation, isocitrate dehydrogenase (IDH) mutation and the co-deletion of chromosome arms $1 \mathrm{p}$ and $19 q(1 p / 19 q)$ have been proven to be strongly associated with the clinical behavior, response to therapy and outcome of HGG [8-10]. Unfortunately, to the best of our knowledge, the relationship between the ME and these molecular alterations has not yet been elucidated.

Therefore, the purpose of the present study was to identify the spatial ME of HGG according to consecutive macropathology, analyze its association with malignant factors including grade, tumor volume $\left(\mathrm{V}_{\text {tumor }}\right)$, location, peritumoral brain edema (PTBE) and molecular markers, and create a model, that could provide evidence for more precisely determining the ME and, hence, the individual CTV to be applied in RT.

\section{Materials And Methods}

\section{Patient selection}

This study involved 30 HGG patients who underwent tumor resection at Shandong Cancer Hospital or the First Affiliated Hospital of Shandong First Medical University between June 2017 and July 2019. The

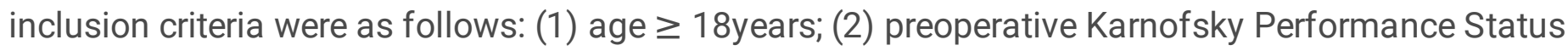
(KPS) $\geq 80$; (3) tumor located in nonfunctional area and successful supra total resection; (4) tumor removal achieved with resection margins that included the neighboring normal tissue (between $2 \mathrm{~cm}$ and $3 \mathrm{~cm}$ away from the tumor border); and (5) no tumor progression observed during follow-up (at least $\geq$ 6 months). The exclusion criteria included: (1) a medical history of brain chemoradiotherapy (CRT) and (2) multicentric or multifocal cerebral lesions. All tumors were graded according to the 2016 World Health Organization (WHO) classification [11]. This study was approved by the institutional review board. All patients provided written informed consent to participate in the trial.

\section{Preoperative MRI Acquisition}


Before any treatment, gadolinium-enhanced MRI examination was performed in all patients. MRI scanning was acquired using a 3T MRI scanner (Philips Achieva 3T). The scanning protocols were obtained with the following parameters. T1-weighted imaging: echo time $(T E)=10 \mathrm{~ms}$, repetition time $(T R)=495 \mathrm{~ms}$, slice thickness $/ \mathrm{gap}=3 \mathrm{~mm} / 0 \mathrm{~mm}$, number of signal averaged $(\mathrm{NSA})=1$, field of view $(\mathrm{FOV})=260 \mathrm{~mm} \times 260 \mathrm{~mm}$, matrix $=256 \times 256$. T2-weighted imaging: $\mathrm{TE}=110 \mathrm{~ms}, \mathrm{TR}=13312 \mathrm{~ms}$, slice thickness $/$ gap $=3 \mathrm{~mm} / 0 \mathrm{~mm}, \mathrm{NSA}=1, \mathrm{FOV}=260 \mathrm{~mm} \times 260 \mathrm{~mm}$, matrix $=416 \times 416$. T2-fluid attenuated inversion recovery $(\mathrm{T} 2-\mathrm{FLAIR}): \mathrm{TE}=120 \mathrm{~ms}, \mathrm{TR}=11000 \mathrm{~ms}$, slice thickness $/ \mathrm{gap}=3 \mathrm{~mm} / 0 \mathrm{~mm}, \mathrm{NSA}=1$, FOV $=260 \mathrm{~mm} \times 260 \mathrm{~mm}$, matrix $=320 \times 320$. To accurately match the MRI and the tissue specimen, the orbitomeatal line $(\mathrm{OML})$ was perpendicular to the scanning table.

Preoperative MRI was evaluated by a senior radiologist and the data (T2-FLAIR and T1-weighted sequence) were used to determine the PTBE volume $\left(\mathrm{V}_{\mathrm{PTBE}}\right), \mathrm{V}_{\text {tumor }}$ and tumor localization. Based on the spatial relationship between the tumor and the subventricular zone (SVZ) and cortex, the tumor location was classified as follows: Type I, tumor contacting SVZ; Type II, tumor involving cortex; Type III, tumor neither contacting SVZ nor infiltrating cortex (Figure 1).

\section{Surgical specimen processing}

After resection, the plane of the OML was marked on the specimen. Subsequently, the surgical specimen was oriented according to the in vivo geometry, marked with different colors to indicate the original orientation of the specimen in the brain and fixed in $10 \%$ formalin $(\geq 24 \mathrm{~h})$. The dimensions of the tumor samples, both before and after fixation were documented to determine the reduction in size due to fixation (Supplementary Table S1). Then, the plane of the OML was perpendicular to the table; the specimen was cut using a tissue slicer from the cranial to the caudal side in approximately $3 \mathrm{~mm}$ thick slices, which ensured that each specimen slice could match the MRI slice. The slices were contiguous, and their individual thickness was measured with a ruler. Finally, whole-mount paraffin sections were made and cut into $5 \mu \mathrm{m}$ sections per slice, which were stained with hematoxylin and eosin (H\&E) (Figures 2a-e). In addition, each patient underwent molecular testing, and the methods used for analyzing the methylation status of the MGMT promoter and determining the mutational status of IDH by DNA pyrosequencing have been described previously $[12,13]$. Deletions of chromosomes $1 p / 19 q$ were evaluated by fluorescence in situ hybridization analysis in tumor tissue sections [12,13].

\section{ME analysis and measurement}

The tumor-containing area and the PTBE area in the H\&E sections were microscopically outlined, scanned and recorded by TissueFAXS PLUS (TissueGnostics, Austria). Subsequently, the scan images were imported into Photoshop (Adobe Systems, USA) to identify the microscopic evidence by two experienced pathologists who were blinded to the clinical data. Invasive GCs were identified by means of their nuclear atypia and heteropyknotic staining [14] (Figure 2f). To measure the spatial distribution of invasive GCs, pathologic slices were used to generate three-dimensional (3D) graphics through 3D-DOCTOR (Able Software Corp, USA). First, the contours of individual H\&E sections were digitized and recorded to 
generate a 3D surface of the reconstructed specimen. Second, the 3D specimens were correct for retraction through scaling parameters (Supplementary Table S1). Then, the corrected 3D image was registered to preoperative T1-weighted MRI using the outline of tumor to perform point-based registration. After the above steps, the ME distance and direction of the GCs relative to the primary tumor bulk were established. In the in-slice direction, the nearest (Euclidean) distance [15] from the edge of the tumor to the microscopic GCs surrounding brain tissue was measured by Photoshop (Figure $2 \mathrm{~g}$ ). In the throughslice direction, the number of slices from the invasive GCs to the lesion border was counted and multiplied by the slice thickness $(\times 3 \mathrm{~mm})$. The ME of each slice is defined as the maximal distance of the ME. The ME of each patient was defined as the maximum ME across different slices (Supplementary Table S2).

\section{Predictive model development and validation}

Based on the multivariate linear regression (MVLR) analysis, we constructed a ME predictive model. To validate the feasibility and safety of this model, we prospectively collected another 30 HGG patients who underwent surgery at Shandong Cancer Hospital between September 2019 and January 2020. The following inclusion criteria were applied: (1) adult patients; (2) preoperative KPS $\geq 70$; and (3) no medical condition that could interfere with oral medication intake. Patients who had a history of a previous cranial surgery, CRT, or contraindication for MRI were excluded. Patients were randomly split into two arms in a 1:1 ratio to receive guideline-based CRT (Group ${ }_{N C C N}$ ) or model-based CRT (Group $\left.{ }_{\text {Model }}\right)$.

All patients started treatment within 2 weeks after surgery and were treated with CRT, which delivered $60 \mathrm{~Gy}$ in 30 fractions with continuous daily temozolomide (TMZ) $\left(75 \mathrm{mg} / \mathrm{m}^{2} / \mathrm{d}\right)$, followed by 6 cycles of adjuvant TMZ (150-200 mg/ $\mathrm{m}^{2}$ for $5 / 28$ days). Radiation treatment planning was performed with the Varian Eclipse Treatment Planning System. Target volumes were delineated according to co-registration of postoperative CT and MRI obtained with each patient in the treatment position. The management of Group $_{\text {NCCN }}$ was designed according to the NCCN guidelines, Version 2.2019. GTV was defined by T1weighted abnormality on the MRI, which consisted of all postoperative-enhanced MRI and the surgical cavity. The CTV was defined by GTV plus a margin of $2 \mathrm{~cm}$, adjusted to anatomical borders. The CTV was expanded by $3 \mathrm{~mm}$ to create the respective planning target volume. For Group ${ }_{\text {Model, }}$ the CTV was defined as the GTV plus a margin, which was determined by our model. The rest of the treatment design was the same as that of Group ${ }_{\mathrm{NCCN}}$.

MRI was repeated before concurrent CRT, before the first cycle of adjuvant TMZ, and thereafter every 26 weeks. Tumor progression and recurrence were identified by both the oncologist and the radiologist. Progression criteria were described by Macdonald et al. [16]. Recurrence patterns were defined as in-field if $\geq 80 \%$ of the lesion resided within the prescription $95 \%$ isodose line $\left(D_{95}\right)$ and marginal if $20-80 \%$ of the recurrence was inside the $D_{95}$. For other cases, recurrences were defined as out-field [17]. The primary endpoint was the overall response rate (ORR) (the proportion of complete response and partial response). Secondary endpoints included progression-free survival (PFS) (the date from surgery to either first 
documented progression or death), and overall survival (OS) (the date from surgery to the date of death or the last follow-up).

\section{Statistical analysis}

For all analyses, we used SPSS 22.0 (IBM Armonk, NY, USA), and values for which $P<0.05$ were considered statistically significant. Categorical variables were expressed as proportions and continuous variables were expressed as mean \pm standard deviation. The difference between two groups was assessed with Student's t-test or Chi-Squared test. When comparing more than two variables, we performed one-way analysis of variance. Post-hoc analysis was used to compare pairwise differences. Spearman's rank correlation was performed to evaluate the relationship of the ME with the grade, $\mathrm{V}_{\text {tumor }}$, location, $\mathrm{V}_{\mathrm{PTBE}}$ and molecular marker status. A MVLR model was created from variables with a $P<0.05$ on correlation analysis, using stepwise regression. To assess the prediction efficiency of this model, calibration was evaluated using the $R$-square goodness-of-fit test, and discrimination was evaluated using receiver operating characteristic (ROC) curves with the corresponding area under the curve (AUC). The patient survival rates were determined by Kaplan-Meier curves and survival curves were analyzed by the log-rank test.

\section{Results}

\section{Patient and tumor characteristics}

In total, $652 \mathrm{H} \& \mathrm{E}$ slides were analyzed in this study. The characteristics of the patients are listed in Table 1. Histological analysis revealed that 17 patients had grade III gliomas (10 anaplastic astrocytomas [AA], 5 anaplastic oligodendrogliomas [AO], and 2 anaplastic oligoastrocytomas [AOA]) and 13 patients had grade IV gliomas (13 glioblastomas [GBM]). The tumor specimen and its radiologic images were almost identical in their morphology. Further analysis revealed that the volume of HGG was similar on T1weighted MRI and in specimens $\left(24.03 \pm 20.54 \mathrm{~cm}^{3}\right.$ vs. $\left.27.14 \pm 22.80 \mathrm{~cm}^{3}, P=0.581\right)$.

\section{Pathologic ME characteristics}

We demonstrated obvious differences in the ME among individuals (Supplementary Table S2). The GCs were heterogeneously distributed through direct invasion, skip metastases, or along neural fiber tracts, pia mater and basement membranes of blood vessels. The mean $M E\left(M E_{\text {mean }}\right)$ distance was $1.70 \mathrm{~cm}$ (range, 0.63 to $2.87 \mathrm{~cm}$ ). The ME distance of $A A, A O, A O A$ and GBM were $1.49 \pm 0.63 \mathrm{~cm}, 1.16 \pm 0.30 \mathrm{~cm}$, $1.47 \pm 0.02 \mathrm{~cm}$ and $2.11 \pm 0.42 \mathrm{~cm}$, respectively. Grade IV gliomas had significantly higher ME than grade III tumors $(2.11 \pm 0.42 \mathrm{~cm}$ vs. $1.39 \pm 0.52 \mathrm{~cm}, P<0.001)$ (Figure 3a). A significant correlation was found between the extent of ME and pathologic grade $(P<0.001)$. However, there was no statistically significant correlation between the extent of ME and $\mathrm{V}_{\text {tumor }}(P=0.779)$ (Table 2$)$.

\section{Relationship between extent of ME and tumor location}


The typical ME distributions in the different locations are shown in Figure 1. Invasive GCs from type I were widely distributed in normal brain tissue, the ME distance was $2.00 \pm 0.57 \mathrm{~cm}$, and in type II, subpial growth became a main pathway for GCs to distant invasion, and the ME distance was $1.97 \pm 0.37 \mathrm{~cm}$; whereas in type III, infiltration occurred in the border of the primary lesion with an ME value of $1.12 \pm$ $0.30 \mathrm{~cm}$. The ME of type I or type II was significantly higher than that of type III (both $P<0.001$ ). However, the ME difference between type I and type II did not reach statistical significance $(P=0.865)$ (Figure 3b). Furthermore, a significant positive correlation was found between the extent of ME and SVZ contact or cortical involvement $(P=0.036$ and 0.044 , respectively) (Table 2).

\section{Relationship between extent of ME and PTBE}

PTBE infiltration was found in all patients. Meanwhile, we observed that GCs invaded beyond the PTBE area in $40 \%(12 / 30)$ of patients, including 7 with perineural spread and 5 with subpial growth (Figures 1ab). In contrast, the invasive GCs from $60 \%$ (18/30) of patients were only contiguous with the lesion (Figure 1c) and showed a much smaller ME range than the PTBE area $\left(24.98 \pm 14.80 \mathrm{~cm}^{3}\right.$ vs. $100.75 \pm$ $\left.52.48 \mathrm{~cm}^{3}, P=0.017\right)$. Spearman's rank correlation analysis revealed no significant relationship between the extent of ME and $\mathrm{V}_{\mathrm{PTBE}}(P=0.751)$ (Table 2).

\section{MGMT, IDH and $1 p / 19 q$ status impact the ME of glioma}

As shown in Figures 3c-e, MGMT methylated tumors had a significantly higher ME than their unmethylated counterparts $(1.90 \pm 0.53 \mathrm{~cm}$ vs. $1.40 \pm 0.57 \mathrm{~cm}, P=0.021)$. In contrast, IDH mutated tumors had a lower ME than IDH wild-type tumors $(1.38 \pm 0.34 \mathrm{~cm}$ vs. $1.91 \pm 0.64 \mathrm{~cm}, P=0.006)$. Tumors with $1 p / 19 q$ co-deletion had a lower ME than those with $1 p / 19 q$ non-co-deletion $(1.39 \pm 0.34 \mathrm{~cm}$ vs. $1.88 \pm$ $0.64 \mathrm{~cm}, P=0.010)$. A significant correlation was found between the extent of ME and MGMT, IDH and $1 p / 19 q$ status $(P=0.022,0.013$ and 0.043 , respectively) (Table 2$)$.

\section{Predictive model analysis and validation}

The MVLR analysis identified that grade, SVZ contact and MGMT, IDH and 1p19q status were independent variables predicting $\mathrm{ME}$ (all $P<0.05$ ), with grade having the largest $\beta$-coefficient $(0.513)$ (Table 3). A predictive model was created as follows: $\mathrm{Y}_{\mathrm{ME}}=0.672+0.513 \mathrm{X}_{\mathrm{Grade}}+0.380 \mathrm{X}_{\mathrm{SVZ}}+$ $0.439 \mathrm{X}_{\mathrm{MGMT}}+0.320 \mathrm{X}_{\mathrm{IDH}}+0.333 \mathrm{X}_{1 \mathrm{p} / 19 \mathrm{q}}$. The model was evaluated with good performance in terms of calibration, with the $R$-square value of the goodness-of-fit test being 0.780 (Figure $4 \mathrm{a}$ ). Meanwhile, we used the $\mathrm{ME}_{\text {mean }}$ value (1.70) as a cutoff to evaluate the discrimination of the model, which proved that the AUC was 0.964 (95\% confidence interval [Cl]: 0.909-1.000, $P<0.001$ ) (Figure $4 \mathrm{~b}$ ).

In the validation cohort (Table 4), Group Model $_{\text {show a smaller CTV volume than Group }}$ NCCN $(67.21 \pm$ $36.98 \mathrm{~cm}^{3}$ vs. $\left.96.25 \pm 37.81 \mathrm{~cm}^{3}, P=0.042\right)$. After a mean follow-up of 7.65 months, 17 patients $(17 / 30$, $56.7 \%)$ had progression or death, which occurred more frequently in Group $\mathrm{NCCN}_{\mathrm{N}}(12 / 15,80 \%)$ than Group $_{\text {Model }}(5 / 15,33.3 \% ; P=0.01)$ (Supplementary Table S3). Group Model $_{\text {had }}$ a higher ORR than 
Group $_{N C C N}(66.7 \%$ vs. $20 \%, P=0.01)$. In Group ${ }_{N C C N}$, recurrence occurred in-field in 2 patients $(40 \%)$, out-

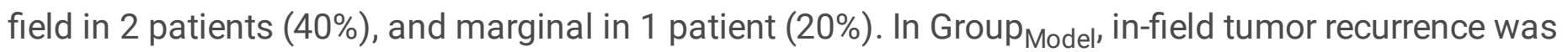
observed in 2 patients $(100 \%)$, and no patients had marginal or out-field recurrence. Group Model $_{\text {patients }}$ had longer PFS than Group ${ }_{N C C N}$ patients $(10.73 \pm 4.15$ months vs. $4.56 \pm 4.50$ months, hazard ratio $[\mathrm{HR}]=$ $0.190,95 \% \mathrm{Cl}, 0.061-0.594, P=0.001$ ) (Figure 4c). However, the OS was $12.52 \pm 2.78$ months in Group $_{\text {Model }}$ patients versus $10.75 \pm 3.97$ months in Group $_{N C C N}$ patients, and this difference was not statistically significant $(\mathrm{HR}=0.175,95 \% \mathrm{Cl}, 0.020-1.501, P=0.072)$ (Figure $4 \mathrm{~d})$.

\section{Discussion}

To our knowledge, this study is the first to determine the CTV margins of HGG based on consecutive macropathology. Our results showed that GC invasion into the surrounding brain tissue is complex and highly heterogeneous across different types of HGGs, according to grade, location and molecular markers. We built and validated an easy-to-use model to guide individualized target delineation.

To date, there is a lack of radiologic-histopathologic correlation studies upon which a consensus can be made to guide the targeted delineation of HGG. Although MRI is proposed as the first choice for pretherapeutic and post-therapeutic evaluation of HGG due to its economic cost-effectiveness and high accuracy, its ability to determine the target volume is inconclusive. Our study found that the contrastenhanced area on T1-weighted MRI reflects only the high-density region of GCs in macropathology, that is, the areas of blood-brain barrier disruption, as described in a previous study [18]. These areas are sufficient for defining the GTV of HGG. However, for CTV delineation, the ability of MRI is limited. Macropathology, as the gold standard of diagnosis, has inherent advantages in evaluating the ME of

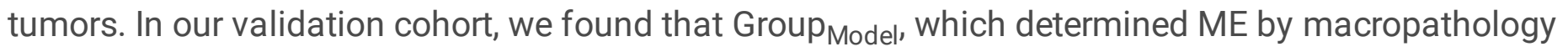
showed significantly higher ORR than Group $_{\mathrm{NCCN}}$, which determined ME by traditional imaging. Meanwhile, Group ${ }_{\text {Model }}$ showed significantly longer PFS than Group $_{\mathrm{NCCN}}$. These findings suggest that model-based RT is effective and feasible, however, a large cohort still needed to further validate our results.

We identified and incorporated 5 independent clinical factors into the MVLR model, including grade, SVZ contact and MGMT, IDH and 1p/19q status. In our model, grade contributed the most to predicting ME of HGG. In agreement with the literature $[13,19,20]$, the WHO grade system is consistently identified as an important factor for ME. We found that higher grade glioma was associated with stronger aggressiveness. Another significant factor influencing the ME on multivariate analysis was preoperative tumor location. It is noteworthy that the invasive GC distribution was wider in tumors contacting the SVZ, which was consistent with the results of previous retrospective studies. Lim et al. [21] demonstrated that SVZ contact was significantly associated with multifocality. In a study by Adeberg et al. [22], glioblastoma that contacted the SVZ showed higher rates of distant progression and multifocal recurrence than noncontacting lesions. This finding may be explained by the recruitment of glioma stemlike cells in the SVZ, resulting in an aggressive glioma subtype [21-23]. In contrast, invasive GCs from type 
III were only contiguous with the lesion. In regard to the study of Adeberg et al. [22], a similar result was found glioblastoma recurrence always occurred in the border of the primary lesion in the tumor, which neither contacted the SVZ nor infiltrated the cortex. Based on these results, the determination of the CTV margin according to different locations has been proposed for the first time, but more evidence is still needed to inform clinical practice.

Further analysis revealed a relationship between the extent of ME and MGMT, IDH and $1 p / 19 q$ status. We found that MGMT methylation induced invasion in distant locations compared with unmethylated cells. Our results confirmed two previous imaging studies $[24,25]$ and showed that methylated glioblastoma patients with MGMT had a greater tendency to develop out-of-field recurrence than those with unmethylated status. Interestingly, the present study also observed that IDH wild-type or 1p/19q non-codeleted patients showed increased tumor migration and invasion compared with their counterparts, which has not been previously reported. These findings might help oncologists provide more tailored RT fields to patients with HGG.

It is highly controversial whether PTBE needs to be intentionally included in the CTV in glioma, since the relationship between the distribution of GCs and PTBE has still been undefined. In the present study, we first comprehensively revealed both relationships through macropathology and found that ME was not significantly associated with PTBE. We observed that the ME range of $60 \%$ of patients was much smaller than that of the PTBE area, whereas infiltration outside of the PTBE occurred when GCs spread along the perineural direction or subpial growth. Similar results were confirmed by Kelly et al. [26] through stereotactic biopsy and Yamahara et al. [27] through autopsy. Based on these important findings, we suggested that RT including the entire PTBE was not necessary. PTBE might merely coexist with infiltrating GCs in what is a spatial coincidence but actually reflects two independent processes; unreasonable RT fields would increase normal tissue toxicity, thereby influencing the prognosis of patients $[28,29]$.

Our study has several limitations. First, only 3 typical molecular prognostic markers were detected, and more genes such as TERT and H3K27M need to be further analyzed to explore biomarkers predicting invasion. Second, the size of our study population was small; thus, a large cohort is needed to further develop and validate our model. Third, our study analyzed only patients with good performance. An inherent bias is that tumors that are amenable to supra total resection likely have anatomic, clinical, or biological characteristics that differ from the majority of tumors where subtotal resection is performed.

In conclusion, tumor cells were heterogeneously distributed in different gliomas. Pathologic grade, location, MGMT, IDH and 1p/19q status were demonstrated to be important factors contributing to ME. This suggested that the delineation of CTV should be individualized. Using these factors, we first built an invasive risk score model, which can better provide valuable evidence to predict the ME of glioma, and this may help clinicians determine the CTV of patients.

\section{Abbreviations}


HGG = High-grade glioma; NCCN = National Comprehensive Cancer Network; CTV = Clinical target volume; GTV = Gross target volume; $\mathrm{CT}=$ Computed tomography; $\mathrm{MRI}=$ Magnetic resonance imaging; $\mathrm{ME}=$ Microscopic extension; $\mathrm{MGMT}=0^{6}$-methylguanine-DNA-methyltransferase; IDH = Isocitrate dehydrogenase; $1 p / 19 q=$ Chromosome arms $1 p$ and $19 q ;$ PTBE $=$ Peritumoral brain edema; KPS = Karnofsky Performance Status; $\mathrm{WHO}=$ World Health Organization; TE = Echo time; $\mathrm{TR}=$ Repetition time; NSA = Number of signal averaged; FOV = Field of view; FLAIR = Fluid attenuated inversion recovery; OML = Orbitomeatal line; SVZ = Subventricular zone; MVLR = Multivariate linear regression; $\mathrm{ORR}=$ Overall response rate; $\mathrm{PFS}=$ Progression-free survival; $\mathrm{OS}=$ Overall survival; $\mathrm{ROC}=$ Receiver operating characteristic; $A A=$ Anaplastic astrocytomas; $A O=$ Anaplastic oligodendrogliomas; $A O A=$ Anaplastic oligoastrocytomas; $\mathrm{Cl}=$ Confidence interval.

\section{Declarations}

\section{Acknowledgements:}

None.

\section{Authors' contributions:}

S-LN was a major contributor in writing the manuscript. Data collection was performed by Y-FZ, JY, TX, SX, J-JS, D-BM, Y-SG and X-CD. Data analyses were performed by X-BZ and Z-QC. J-MY and MH designed the work and provided the financial support. J-MY and $\mathrm{MH}$ revised the manuscript. All authors contributed to the article and approved the final manuscript.

\section{Funding:}

This work was supported by National Key Research and Development Program of China (No. 2018YFE0114100), Key Research and Development Program of Shandong province, China (No. 2019GGX101057) and Science Technology Program of Jinan (No. 201805051).

\section{Availability of data and materials:}

The data used in this analysis is from publications available in the public domain.

\section{Ethics approval and consent to participate:}

This study was approved by the institutional review board. All patients provided written informed consent to participate in the trial.

\section{Consent for publication:}

Not applicable.

Competing interests: 
The authors declare that they have no competing interests.

\section{Footnotes:}

Data were presented in part at the 62nd Annual Meeting of the American Society for Radiation Oncology (ASTRO), Miami, Florida, USA, October 25-28, 2020 (Quick Pitch Oral). The abstract in current study was designated as a 2020 Best of ASTRO abstract.

\section{References}

1. Niyazi M, Brada M, Chalmers AJ, et al. Estro-acrop guideline "target delineation of glioblastomas". Radiother Oncol 2016;118:35-42.

2. Aydin $\mathrm{H}$, Sillenberg I, von Lieven $\mathrm{H}$. Patterns of failure following ct-based 3-d irradiation for malignant glioma. Strahlenther Onkol 2001;177:424-31.

3. Wallner KE, Galicich JH, Krol G, et al. Patterns of failure following treatment for glioblastoma multiforme and anaplastic astrocytoma. Int J Radiat Oncol Biol Phys 1989;16:1405-9.

4. Chang EL, Akyurek S, Avalos T, et al. Evaluation of peritumoral edema in the delineation of RT clinical target volumes for glioblastoma. Int J Radiat Oncol Biol Phys 2007;68:144-50.

5. Burger PC, Heinz ER, Shibata T, et al. Topographic anatomy and ct correlations in the untreated glioblastoma multiforme. J Neurosurg 1988;68:698-704.

6. Johnson PC, Hunt SJ, Drayer BP. Human cerebral gliomas: Correlation of postmortem mr imaging and neuropathologic findings. Radiology 1989;170:211-7.

7. Kelly PJ, Daumas-Duport C, Kispert DB, et al. Imaging-based stereotaxic serial biopsies in untreated intracranial glial neoplasms. J Neurosurg 1987;66:865-74.

8. Perry JR, Laperriere N, O'Callaghan CJ, et al. Short-course radiation plus temozolomide in elderly patients with glioblastoma. N Engl J Med 2017;376:1027-1037.

9. Baumert BG, Hegi ME, van den Bent MJ, et al. Temozolomide chemotherapy versus RT in high-risk low-grade glioma (eortc 22033-26033): A randomised, open-label, phase 3 intergroup study. Lancet Oncol 2016;17:1521-1532.

10. Aldape K, Zadeh G, Mansouri S, et al. Glioblastoma: Pathology, molecular mechanisms and markers. Acta Neuropathol 2015;129:829-48.

11. Louis DN, Perry A, Reifenberger G, et al. The 2016 world health organization classification of tumors of the central nervous system: A summary. Acta Neuropathol 2016;131:803-20.

12. Yang P, Cai J, Yan W, et al. Classification based on mutations of tert promoter and idh characterizes subtypes in grade ii/iii gliomas. Neuro Oncol 2016;18:1099-108.

13. Fan Z, Liu Y, Li S, et al. Association of tumor growth rates with molecular biomarker status: A longitudinal study of high-grade glioma. Aging (Albany NY) 2020;12:7908-7926.

14. Mangiola A, de Bonis $P$, Maira $G$, et al. Invasive tumor cells and prognosis in a selected population of patients with glioblastoma multiforme. Cancer 2008;113:841-6. 
15. Apparicio P, Gelb J, Dube AS, et al. The approaches to measuring the potential spatial access to urban health services revisited: Distance types and aggregation-error issues. Int J Health Geogr 2017;16:32.

16. Macdonald DR, Cascino TL, Schold SC, Jr., et al. Response criteria for phase ii studies of supratentorial malignant glioma. J Clin Oncol 1990;8:1277-80.

17. Lee SW, Fraass BA, Marsh LH, et al. Patterns of failure following high-dose 3-d conformal radiotherapy for high-grade astrocytomas: A quantitative dosimetric study. Int J Radiat Oncol Biol Phys 1999;43:79-88.

18. Whitfield GA, Kennedy SR, Djoukhadar IK, et al. Imaging and target volume delineation in glioma. Clin Oncol (R Coll Radiol) 2014;26:364-76.

19. Cuddapah VA, Robel S, Watkins $S$, et al. A neurocentric perspective on glioma invasion. Nat Rev Neurosci 2014;15:455-65.

20. Louis DN. Molecular pathology of malignant gliomas. Annu Rev Pathol 2006;1:97-117.

21. Lim DA, Cha S, Mayo MC, et al. Relationship of glioblastoma multiforme to neural stem cell regions predicts invasive and multifocal tumor phenotype. Neuro Oncol 2007;9:424-9.

22. Adeberg S, Konig L, Bostel T, et al. Glioblastoma recurrence patterns after radiation therapy with regard to the subventricular zone. Int J Radiat Oncol Biol Phys 2014;90:886-93.

23. Chen L, Chaichana KL, Kleinberg L, et al. Glioblastoma recurrence patterns near neural stem cell regions. Radiother Oncol 2015;116:294-300.

24. Brandes AA, Tosoni A, Franceschi E, et al. Recurrence pattern after temozolomide concomitant with and adjuvant to RT in newly diagnosed patients with glioblastoma: Correlation with mgmt promoter methylation status. J Clin Oncol 2009;27:1275-9.

25. Minniti G, Amelio D, Amichetti $M$, et al. Patterns of failure and comparison of different target volume delineations in patients with glioblastoma treated with conformal RT plus concomitant and adjuvant temozolomide. Radiother Oncol 2010;97:377-81.

26. Kelly PJ, Daumas-Duport C, Scheithauer BW, et al. Stereotactic histologic correlations of computed tomography- and magnetic resonance imaging-defined abnormalities in patients with glial neoplasms. Mayo Clin Proc 1987;62:450-9.

27. Yamahara T, Numa Y, Oishi T, et al. Morphological and flow cytometric analysis of cell infiltration in glioblastoma: A comparison of autopsy brain and neuroimaging. Brain Tumor Pathol 2010;27:81-7.

28. Giese A. Glioma invasion-pattern of dissemination by mechanisms of invasion and surgical intervention, pattern of gene expression and its regulatory control by tumorsuppressor p53 and protooncogene ets-1. Acta Neurochir Suppl 2003;88:153-62.

29. Chang EL, Akyurek S, Avalos T, et al. Evaluation of peritumoral edema in the delineation of RT clinical target volumes for glioblastoma. Int J Radiat Oncol Biol Phys 2007;68:144-50.

\section{Tables}


Table 1

Baseline characteristics of HGG patients

\begin{tabular}{|c|c|c|}
\hline Characteristic & No. of patients & $\%$ \\
\hline \multicolumn{3}{|l|}{ Gender } \\
\hline Male & 16 & 53.3 \\
\hline Female & 14 & 46.7 \\
\hline \multicolumn{3}{|l|}{ Age (years) } \\
\hline$\leq 50$ & 17 & 56.7 \\
\hline$>50$ & 13 & 43.3 \\
\hline \multicolumn{3}{|c|}{ Preoperative KPS } \\
\hline $80-90$ & 8 & 26.7 \\
\hline$\geq 90$ & 22 & 73.3 \\
\hline \multicolumn{3}{|c|}{ Pathologic grade } \\
\hline Grade III & 17 & 56.7 \\
\hline Grade IV & 13 & 43.3 \\
\hline \multicolumn{3}{|l|}{ Tumor location } \\
\hline Type I & 11 & 36.7 \\
\hline Type II & 9 & 30 \\
\hline Type III & 10 & 33.3 \\
\hline \multicolumn{3}{|c|}{ Molecular markers } \\
\hline \multicolumn{3}{|c|}{ MGMT methylation status } \\
\hline Unmethylated & 12 & 40 \\
\hline Methylated & 18 & 60 \\
\hline \multicolumn{3}{|c|}{ IDH mutation status } \\
\hline Mutated & 12 & 40 \\
\hline Wild type & 18 & 60 \\
\hline \multicolumn{3}{|c|}{$1 p / 19 q$ co-deletion status } \\
\hline Co-deleted & 11 & 36.7 \\
\hline Non-co-deleted & 19 & 63.3 \\
\hline
\end{tabular}




\begin{tabular}{|lll|}
\hline AA & 10 & 33.3 \\
\hline AO & 5 & 16.7 \\
\hline AOA & 2 & 6.7 \\
\hline GBM & 13 & 43.3 \\
\hline
\end{tabular}

Abbreviation: $\mathrm{HGG}=$ high-grade glioma; $\mathrm{KPS}=$ Karnofsky performance status; $\mathrm{MGMT}=0^{6}$ methylguanine-DNA-methyltransferase; IDH = isocitrate dehydrogenase; $1 \mathrm{p} / 19 \mathrm{q}$ co-deletion $=$ the codeletion of chromosome arms $1 p$ and $19 q ; A A=$ anaplastic astrocytoma; $A O=$ anaplastic oligodendrogliomas; $\mathrm{AOA}$ = anaplastic oligoastrocytomas; $\mathrm{GBM}=$ glioblastoma

Table 2

Correlation between ME extent and the different variables

\begin{tabular}{|lll|}
\hline Variables & \multicolumn{2}{l|}{ ME extent } \\
\cline { 2 - 3 } & $r$ value & $P$ value $^{\star}$ \\
\hline Pathologic grade & & \\
\hline III/IV & 0.668 & $<0.001$ \\
\hline Tumor volume & 0.053 & 0.779 \\
\hline SVZ contact & & \\
\hline No/Yes & 0.384 & $\mathbf{0 . 0 3 6}$ \\
\hline Cortical involvement & & \\
\hline No/Yes & 0.370 & $\mathbf{0 . 0 4 4}$ \\
\hline PTBE volume & 0.061 & 0.751 \\
\hline MGMT methylation status & & \\
\hline Unmethylated/Methylated & 0.417 & $\mathbf{0 . 0 2 2}$ \\
\hline IDH mutation status & & \\
\hline Mutated/Wild type & 0.448 & $\mathbf{0 . 0 1 3}$ \\
\hline 1p/19q co-deletion status & & \\
\hline Co-deleted/Non-co-deleted & 0.372 & $\mathbf{0 . 0 4 3}$ \\
\hline
\end{tabular}

${ }^{*} r$ and $P$ value according to the spearman's rank correlation. 
Abbreviation: $\mathrm{ME}$ = microscopic extension; $\mathrm{SVZ}$ = subventricular zone; $\mathrm{PTBE}$ = peritumoral brain edema; MGMT = $0^{6}$-methylguanine-DNA-methyltransferase; IDH = isocitrate dehydrogenase; $1 \mathrm{p} / 19 \mathrm{q}$ co-deletion = the co-deletion of chromosome arms $1 p$ and $19 q$

Table 3

Variables associated with ME extent in multivariate linear regression model

\begin{tabular}{|c|c|c|c|c|c|}
\hline Variable & $\beta$ & SE & $95 \% \mathrm{Cl}$ for $\beta$ & $P$ value & $\begin{array}{c}\text { Score } \\
(0,1)\end{array}$ \\
\hline Constant & 0.672 & 0.139 & $0.385-0.958$ & $<0.001$ & \\
\hline Pathologic grade & 0.513 & 0.136 & $0.232-0.794$ & 0.001 & \\
\hline Grade III & & & & & 0 \\
\hline Grade IV & & & & & 1 \\
\hline SVZ contact & 0.380 & 0.124 & $0.125-0.635$ & 0.005 & \\
\hline No & & & & & 0 \\
\hline Yes & & & & & 1 \\
\hline \multicolumn{6}{|l|}{ Molecular markers } \\
\hline MGMT methylation status & 0.439 & 0.129 & $0.171-0.706$ & 0.002 & \\
\hline Unmethylated & & & & & 0 \\
\hline Methylated & & & & & 1 \\
\hline IDH mutation status & 0.320 & 0.141 & $0.028-0.611$ & 0.033 & \\
\hline Mutated & & & & & 0 \\
\hline Wild type & & & & & 1 \\
\hline 1p/19q co-deletion status & 0.333 & 0.120 & $0.084-0.581$ & 0.011 & \\
\hline Co-deleted & & & & & 0 \\
\hline Non-co-deleted & & & & & 1 \\
\hline
\end{tabular}

Abbreviation: $\mathrm{ME}=$ microscopic extension; $\beta$ = regression coefficient; $\mathrm{SE}=$ standard error; $\mathrm{Cl}=$ confidence interval; SVZ = subventricular zone; MGMT = $0^{6}$-methylguanine-DNA-methyltransferase; IDH = isocitrate dehydrogenase; $1 p / 19 q$ co-deletion $=$ the co-deletion of chromosome arms $1 p$ and $19 q$ 
Table 4

Baseline characteristics of validation cohort

\begin{tabular}{|c|c|c|c|}
\hline Characteristic & $\begin{array}{l}\text { Group }_{\mathrm{NCCN}} \\
\text { no. }(\%)\end{array}$ & $\begin{array}{l}\text { Group }_{\text {Model }} \\
\text { no. }(\%)\end{array}$ & $P$ value \\
\hline Sex & & & 0.136 \\
\hline Male & $11(73.3)$ & $7(46.7)$ & \\
\hline Female & $4(26.7)$ & $8(53.3)$ & \\
\hline Age (years) & & & 0.464 \\
\hline$\leq 50$ & $6(40)$ & $8(53.3)$ & \\
\hline$>50$ & $9(60)$ & $7(46.7)$ & \\
\hline Preoperative KPS & & & 0.624 \\
\hline$\geq 80$ & $13(86.7)$ & $12(80)$ & \\
\hline$<80$ & $2(13.3)$ & $3(20)$ & \\
\hline Extent of resection & & & 0.361 \\
\hline Gross total resection & $11(73.3)$ & $13(86.7)$ & \\
\hline Subtotal resection & $4(26.7)$ & $2(13.3)$ & \\
\hline Tumor location & & & 0.621 \\
\hline Type I & $5(33.3)$ & $5(33.3)$ & \\
\hline Type II & $6(40)$ & $8(53.3)$ & \\
\hline Type III & $4(26.7)$ & $2(13.3)$ & \\
\hline Pathologic grade & & & 0.690 \\
\hline Grade III & $4(26.7)$ & $5(33.3)$ & \\
\hline Grade IV & $11(73.3)$ & $10(66.7)$ & \\
\hline \multicolumn{4}{|l|}{ Molecular markers } \\
\hline MGMT methylation status & & & 0.439 \\
\hline Unmethylated & $11(73.3)$ & $9(60)$ & \\
\hline Methylated & $4(26.7)$ & $6(40)$ & \\
\hline IDH mutation status & & & 0.409 \\
\hline Mutated & $5(33.3)$ & $3(20)$ & \\
\hline Wild type & $10(66.7)$ & $12(80)$ & \\
\hline
\end{tabular}




\begin{tabular}{|llll|}
\hline 1p/19q co-deletion status & & & 1.00 \\
\hline Co-deleted & $6(40)$ & $6(40)$ & \\
\hline Non-co-deleted & $9(60)$ & $9(60)$ & \\
\hline Histology & & & 0.563 \\
\hline AA & $2(13.3)$ & $2(13.3)$ & \\
AO & $1(6.7)$ & $3(20)$ \\
AOA & $1(6.7)$ & 0 \\
\hline GBM & $11(73.3)$ & $10(66.7)$ \\
\hline
\end{tabular}

${ }^{*}$ P value according to the Chi-Squared test.

Abbreviations: NCCN = National Comprehensive Cancer Network; KPS = Karnofsky Performance Status; MGMT = $0^{6}$-methylguanine-DNA-methyltransferase; IDH = isocitrate dehydrogenase; $1 \mathrm{p} / 19 \mathrm{q}$ co-deletion = the co-deletion of chromosome arms $1 p$ and $19 q ; A A=$ anaplastic astrocytoma; $A O=$ anaplastic oligodendrogliomas; $\mathrm{AOA}$ = anaplastic oligoastrocytomas; $\mathrm{GBM}$ = glioblastoma

\section{Figures}




\section{Fig. 1}
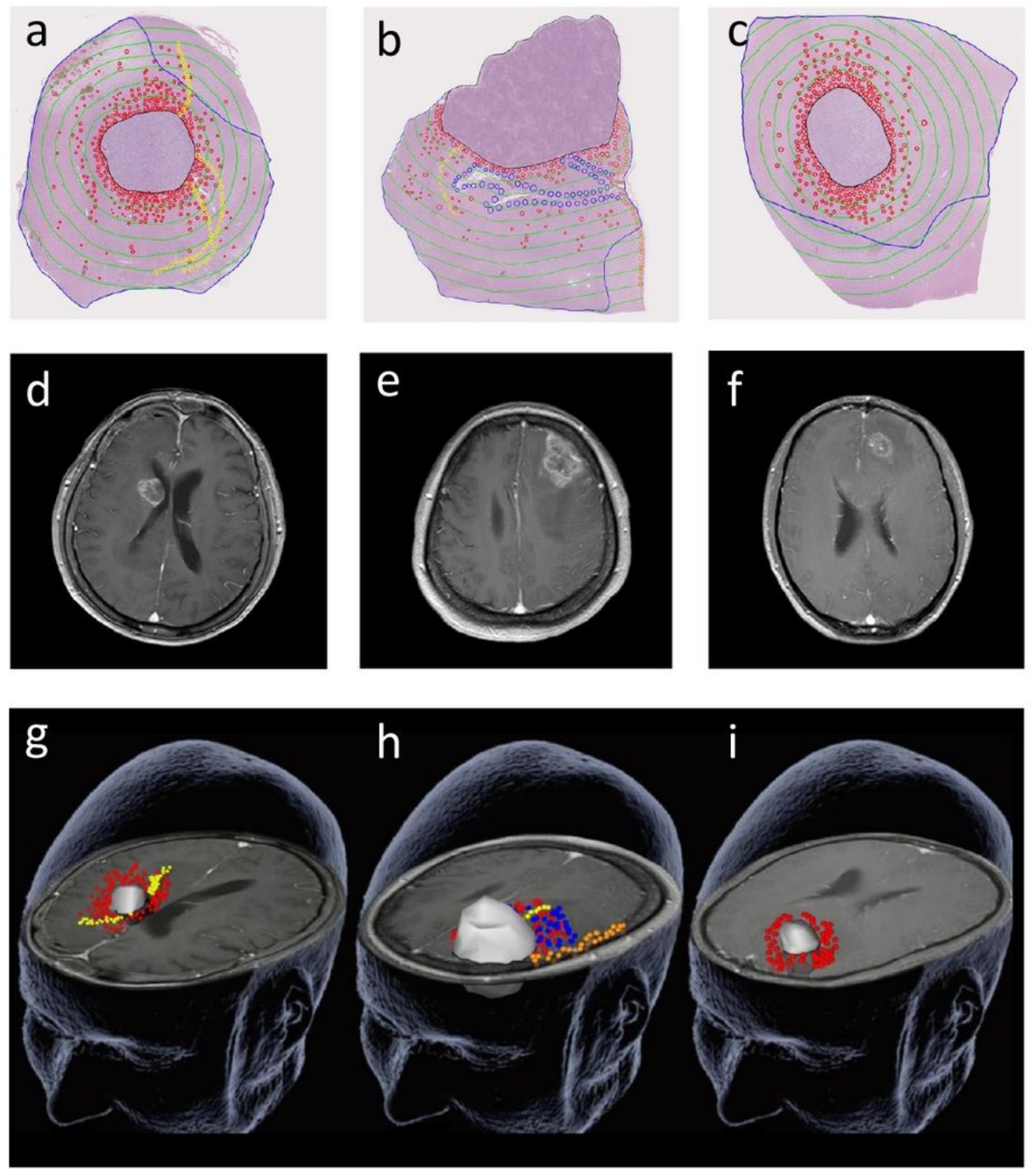

\section{Figure 1}

Tumor microscopic extension distribution for different locations based on preoperative T1-weighted MRI. $(a, d, g)$ Type I; (b, e, h) Type Il; (c, f, i) Type III. At the sites where the glioma cells produced direct invasion, perineural spread, subpial growth and perivascular spread are outlined in red, yellow, orange and blue, respectively. Black line: tumor-containing area; blue line: PTBE-containing area; green line: $0.25 \mathrm{~cm}$ intervals. 


\section{Fig. 2}
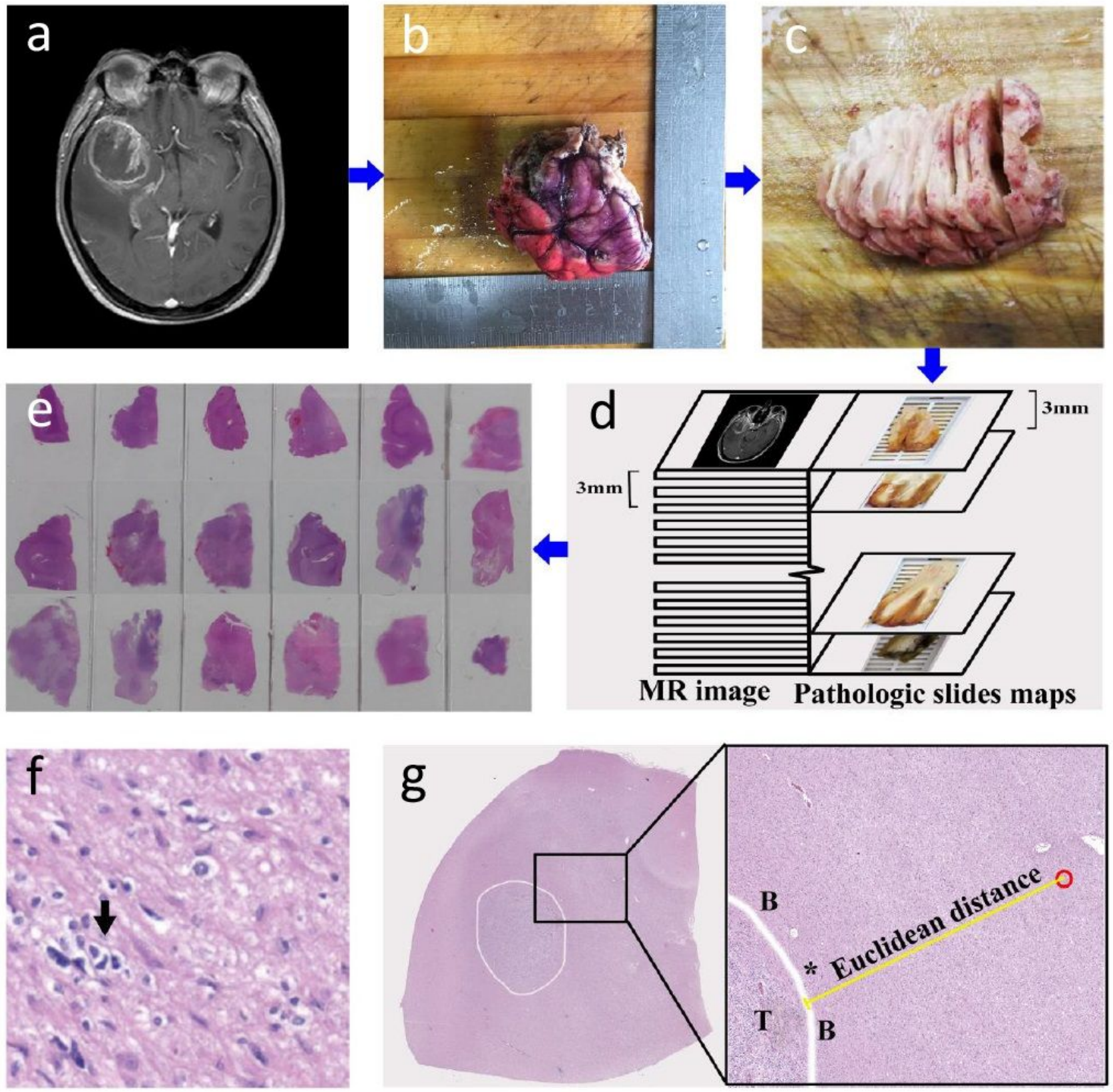

\section{Figure 2}

(a-e) Pathology procedure. First, by referring to MRI (a), the surgical specimen was oriented according to the in vivo geometry, and the colored edges indicate the direction of the specimen in the brain (b). Afterward, the fixed specimen was sectioned consecutively at approximately $3.00 \mathrm{~mm}$ intervals (c), which ensured that each specimen slice matched the MRI slice (d). Finally, the tissue specimen was selected for $H \& E$ (e). (f) Histologic features of areas surrounding the glioma: An atypical cell with a moderately irregular nucleus suspected of neoplasia (arrow) is shown. (g) H\&E staining cross-section (5×magnification) shows a wide view of the specimen tissue section with the tumor outlined in white. 
Magnified H\&E view (10xmagnification) shows invasive glioma cells (outlined in red) outside the boundary $(\mathrm{B})$ of the tumor $(\mathrm{T})$. The ME (Euclidean) distance from the edge of the tumor ${ }^{*}$ ) to the microscopic tumor cells surrounding brain tissue was measured (yellow line).

Fig. 3


Preoperative tumor location
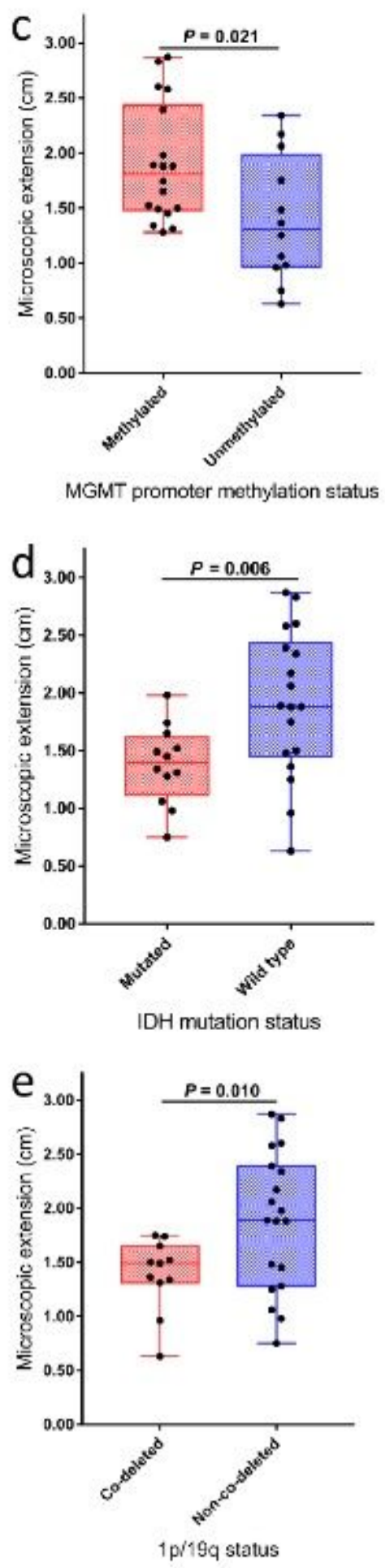

Figure 3

Box plot analysis showing the microscopic extension in different subgroups. (a) Pathologic grade; (b) preoperative tumor location; (c) MGMT promoter methylation status; (d) IDH mutation status; (e) 1p/19q 
co-deletion status.

Fig. 4
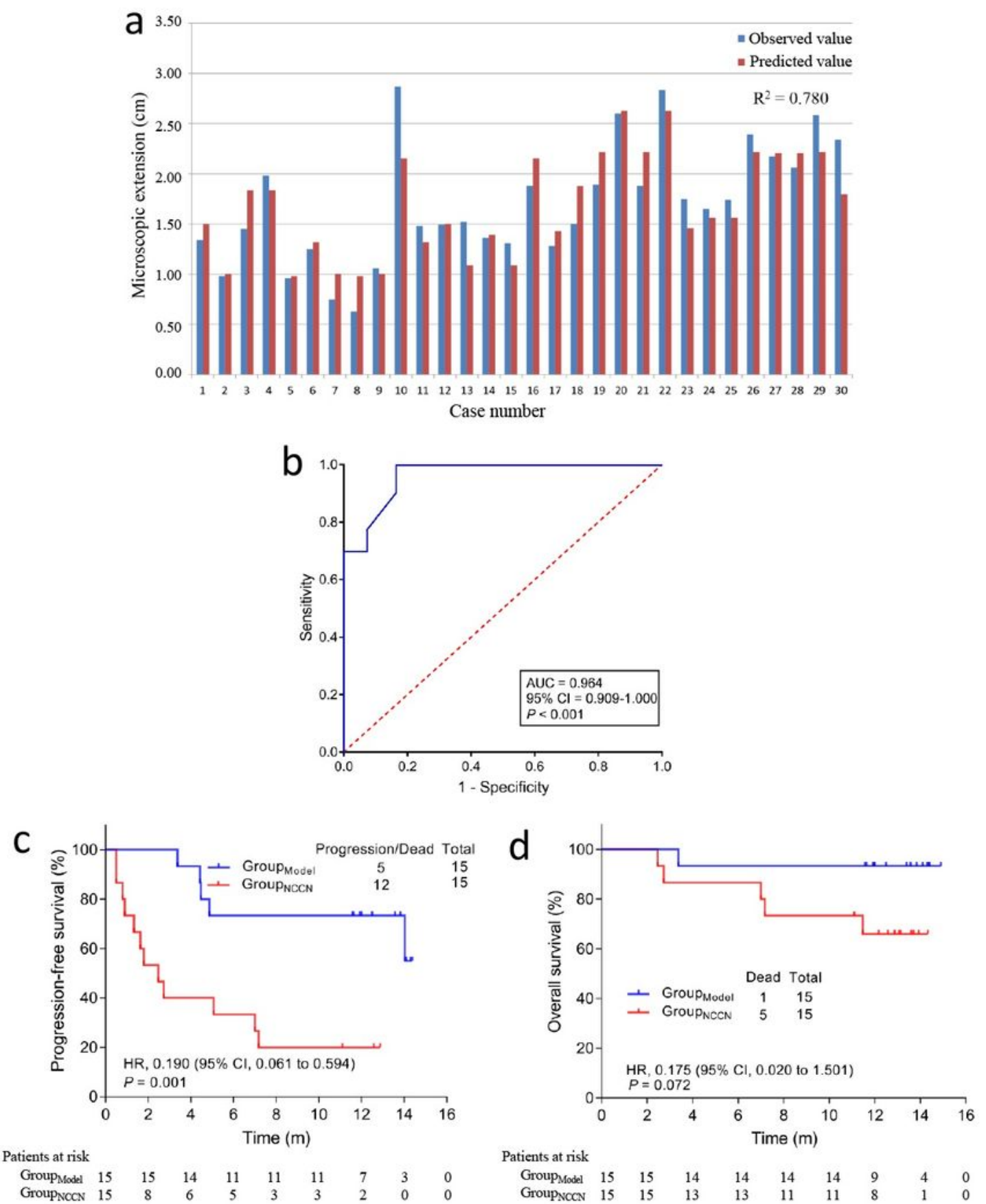

Patients at risk

$\begin{array}{llllllllll}\text { Group }_{\text {Model }} & 15 & 15 & 14 & 14 & 14 & 14 & 9 & 4 & 0 \\ \text { Group }_{\text {NoCN }} & 15 & 15 & 13 & 13 & 11 & 11 & 8 & 1 & 0\end{array}$

\section{Figure 4}

(a) Histogram plot showing the calibration of the predictive model; (b) ROC curves depicting the predictive discrimination of the model; (c) Kaplan-Meier estimates of progression-free survival by validation cohort; (d) Kaplan-Meier estimates of overall survival by validation cohort. 


\section{Supplementary Files}

This is a list of supplementary files associated with this preprint. Click to download.

- SupplementaryMaterial.docx 